\title{
Conceptualization of Persian Relative Structure in Cognitive Grammar
}

\author{
Dr.Arsalan Golfam \\ Department of Linguistics,Tarbiat Modarres University,Tehran,Iran. \\ golfamarsalan@gmail.com \\ Dr.Alieh Kord Zaferanloo Kamboozia \\ Department of Linguistics,Tarbiat Modarres University,Tehran,Iran. \\ Akord@modares.ac.ir \\ Dr.Ferdows Aghagolzadeh \\ Department of Linguistics,Tarbiat Modarres University,Tehran,Iran. \\ Aghagolz@modares.ac.ir \\ Sahel Khoshbakht Ghahfarrokhi \\ Department of Linguistics,Tarbiat Modarres University,Tehran,Iran. \\ sahel.khoshbakht@yahoo.com
}

\begin{abstract}
In the cognitive approach to linguistics, language is considered as a part of the cognitive system which mirrors the conceptual organization as well as the world within the speakers' mind. This modern approach includes a variety of principles, assumptions and models, among which Cognitive Grammar Model is recognized as the most noticeable one. This grammar considers a symbolic nature for the language which symbolizes the meaning and thought. On the other hand, relativization as a notion in every individual's cognition is considered as a universal manifested in all languages. Thus, the present research tries to clarify the conceptualization and symbolization of Persian relative structure by the use of cognitive means. It, also studies different types of relative structures in Persian, on the basis of Langacker (2008) model and differentiates the restrictive and non-restrictive relative clauses in Persian. The method used in the analysis of this research is the descriptive-analytical method. For the data collection, the corpus method is used and examples of Persian relative structures are studied. The present research findings show that the conceptualization of Persian relative structures can be clarified in the cognitive approach and Persian relative structures can be studied on the basis of Langacker model.
\end{abstract}

Keywords: Cognitive Linguistics, Cognitive Grammar, Relativization, Relative clause, Restrictive, Nonrestrictive

\section{Academic Discipline And Sub-Disciplines}

Linguistics

\section{SUBJECT CLASSIFICATION}

Cognitive linguistics

\section{METHOD}

Descriptive-Analytical method

\section{INTRODUCTION}

The purpose of this article is to explain the conceptualization of relative structures and to study these structures in Persian language from the CG(Cognitive Grammar) point of view. In cognitive linguistics, it is believed that the experience of the outside world and cognition are mirrored in both the structure and role of language and the structure of the language reflects the conceptual structure. According to CG, linguistic units are all symbolic and symbolize conceptual content, hence the language has a symbolic structure. Since CG is a modern domain, few linguistic structures in Persian have been studied from this perspective. Among the linguistic structures in Persian, relative structures can be mentioned. The relative clause in Persian language, appears as the last part of the naminal group and describes it. In general, the concept of relativization can be regarded as one of the most basic concepts of the human mind. According to Whaley (1997), the ability to put a clause in other structures, is one of the features of the human language, with which human can produce infinitely long sentences.

Therefore, the present article, as the first research in Persian language studies, is an attempt to clarify the conceptualization and symbolization of Persian relative structure by using specific means and concepts of the cognitive approach, and seeks to answer whether it is possible to study the different Persian relative structures in Langacker model (2008) and how Persian restrictive and non- restrictive relative clauses can be distinguished in CG. 


\section{LITERATURE REVIEW}

Comrie (1981) studies the relative clauses in a typological approach. He divides the relative clauses to restrictive and nonrestrictive and believes that they are radically different in semantic or pragmatic terms, in particular, the restrictive relative clause uses presupposed information to identify the referent of a noun phrase, while the non- restrictive relative clause presents new information on the basis of the assumption that the referent can already be identified. He also mentions that in some languages like Persian, this distinction is formally realized too. He assumes that the restrictive relative clauses are more central to the notion of relative clause than are the non- restrictive relative clauses so he writes that a relative clause consists necessarily of a head and a restricting clause. The head in itself, has a certain potential range of referents, but the restricting clause restricts this set, by giving a proposition that must be true of the actual referents of the over-all construction.

Whaley (1997) finds the relative clauses as modifiers of nouns like adjective clauses. He calls the noun being modified by the relative clause as the head noun and the word or particle introducing the relative clause as relativizer or relative pronoun. Whaley, also divides the relative clauses to restrictive and non- restrictive , on the basis of the semantic relationship that the relative clauses have to the nouns they modify. He believes that the ability to embed clauses within other structures is one of the hallmarks of human language and by virtue of this ability, which is shared by all languages, speakers can, in principle, generate infinitely long sentences.

Murcia and Freeman (1999: 579) argue that relative clauses provide the necessary means for encoding complex descriptors, which use less words than the two independent clauses do. They divide the relative clauses, on the base of the syntactic role of the head noun. In the relative structure, the head noun has two roles; a role in the main clause and the same or different role in the relative clause. According to the above definition, four types of relative clauses are considered: 1. Subject-Subject (SS). 2. Subject-Object (SO). 3. Object-Object (OO) clause 4. Object-Subject (OS)(Murcia \& Freeman, 1999: 577).

Gholam AliZadeh (2001: 155-159) defines relative clause as a dependent clause which modifies the noun phrase. There are two types of relative clauses In Persian: a restrictive and a non-restrictive relative clause. One difference between these two types of clauses is that one [i] appears at the end of noun phrase modified by the restrictive clause, and there is neither at the end of this clause nor in the beginning of it ,that a pause appears. On the other hand, at the end of the noun phrase modified by non-restrictive clause, [i] does not appear, but there is a pause both in the beginning and at the end of the clause. Another important difference between these two clauses is the possibility to exclude non-restrictive clause, without damaging the syntactic structure. However, the presence of a restrictive clause is necessary to specify the scope and the semantic domain of the noun modified.

Langacker (2008) believes that whereas an adverbial clause modifies another clause, a relative clause modifies a nominal expression. The primary connection is thus a correspondence between the nominal referent and some participant in the process designated by the relative. That participant, sometimes called the pivot, has a semantic role in both the relative clause and the matrix(main) clause containing the modified nominal. He also mentions that a relative construction has two components: a nominal expression that specifies a basic type, and a clause which helps identify a particular instance of that type. They are integrated to form a higher-level nominal through a correspondence between the nominal profile and a schematic clausal participant. The essence of relative clause constructions does not consist, however, in any specific structural configuration. Their essential feature is semantic: a relative clause is one invoked to characterize a nominal referent identified as a participant in the clausal process.

\section{FRAMEWORK:COGNITIVE GRAMMAR}

Cognitive Grammar belongs to the wider movement known as cognitive linguistics, which in turn is part of the functional tradition. This framework offers a comprehensive yet coherent view of language structure, with the further advantages of being intuitively natural, psychologically plausible, and empirically viable. CG claims that grammar is meaningful. On the other hand, CG's most fundamental claim is that grammar is symbolic in nature; A symbol as the pairing between a semantic structure and a phonological structure, such that one is able to evoke the other. Thus, grammar allows us to construct and symbolize the more elaborate meanings of complex expressions (like phrases, clauses, and sentences). It is then an essential aspect of the conceptual apparatus through which we apprehend and engage the world. Additionally, instead of being a distinct and self-contained cognitive system, grammar is not only an integral part of cognition but also a key to understanding it. Therefore, it is more reasonable to suppose that grammar, rather than being separate and distinct, is merely the abstract commonality inherent in sets of symbolically complex expressions.(langacker,2008)

\subsection{Langacker Model}

\subsubsection{Relative Clauses}

As mentioned before Langacker (2008,423-429) believes that when a relative clause modifies a nominal expression, the primary connection is a correspondence between the nominal referent and some participant in the process designated by the relative. That participant has a semantic role in both the relative clause and the matrix clause containing the modified nominal. In (1), for example, the relative clause "I was reading" modifies "book". The book referred to is the pivot. It is understood as both trajectory of the matrix clause, where it functions overtly as the subject, and as landmark of the relative.

(1) The book I was reading offended her. 
The essential correspondence equates the profile of "the book" with the schematic landmark of "I was reading". The former being the profile determinant (constructional head), the composite expression designates "the book" rather than the process of reading it. The result is a complex nominal, "the book I was reading", which specifies the trajector of offended her at a higher level of organization. Hence the topmost structure, representing the composite meaning for the sentence as a whole, shows the book as having two roles: it participates in both the offending (profiled at that level) and the reading.

\subsubsection{Restrictive and Non- Restrictive Relative Clauses}

According to Langacker (2008) if a modifier plays no role in identifying the nominal referent, it will be a departure from the canon. A well-known case is the non-restrictive relative clauses. A restrictive relative clause serves to limit the pool of eligible candidates, restricting it to a subset of the basic type's maximal extension. In (2)(a)-where these candidates really are candidates - the specified property (really deserving to win) limits the pool to a single candidate, as required by the definite article:

\section{(2) (a) The candidate who really deserves to win ran a positive campaign. \\ (b) The candidate, who really deserves to win, ran a positive campaign.}

The information supplied by a non-restrictive clause fails to be exploited in this manner. In (2)(b), the profiled instance of candidate is contextually identified independently of deserving to win (rather than on the basis of that property). To represent the distinction, restrictive relative clauses are usually analyzed as being part of the nominal in question, and non-restrictive clauses as being external to it. Supporting this analysis are the pauses ("comma intonation") associated with non- restrictive relatives. In and of itself, however, this structural difference is not sufficient to account for the semantic contrast. While they are usually contiguous, a non-restrictive relative is set off from the nominal component by the slight hesitation written as a comma. This prosodic separation is an indication that they occupy separate windows of attention, and are thus in large measure apprehended independently. This is possible because a non- restrictive relative is not invoked to single out the nominal referent, but to make an additional comment about it.

\section{DATA ANALYSIS}

The method used in the analysis of this paper is a descriptive and analytical linguistic method. In this way, first, the conceptualization of Persian relative structure is examined and then the symbolizing of relative structure is discussed, Subsequently, in the following two sub-sections, based on Langacker(2008) model, different Persian relative structures are studied and the grounding of the head noun is examined in CG. For data collection, the corpus method is used and examples of Persian relative structures are investigated.

\subsection{Conceptualization of Relative Structure}

Since the capability to conceptualize the relative structure exists in each individual cognition, the cognitive approach means and concepts, including the schema, can be used to clarify that. The schema are the conceptual structures in people cognition which they use to conceptualize the experience of the world around them, and to create linguistic structures(Safavi,2008). Among the existing schema, containment schema play an important role in the conceptualization of Persian relative structures.

In Persian, each relative structure is composed of four general parts: the matrix clause, the dependent clause or the relative clause, the head noun, and the morpheme "ke" at the beginning of relative clause, which is in fact , a link between the matrix and the relative clause. Therefore, in Persian, the relative clause appears after the head noun and modifies it, and the matrix clause becomes an enveloping sentence that enfolds the relative clause, and ultimately from this combination, relative structure is formed.

As mentioned above, it seems Persian speaker, when confronted with a relative situation and wants to describe a subject, which itself is a part of a larger event called the matrix clause, uses a different event called relative clause, and by considering the matrix clause event as a container, places that relative clause within it, and conceptualizes the relative structure. Look at the following example:

\section{(3) Pan mærdi ke be to komæk kærd pedære mæn ? ?æst.}

$\begin{array}{lll}\text { 1stpsing (First person singular) past } & \text { 3rdpsing (Third person singular) present,3rdpsing } \\ \text { That man who you helped father my is. }\end{array}$

That man who helped you is my father.

\section{Subject: That man}

Matrix event: That man is my father.

Relative event: (That man) helped you.

In example(3), to modify the subject "That man" which is a part of matrix event, the relative event is used and this event is placed inside the matrix event. As shown in Figure(1).

Therefore, it can be claimed that Persian relative structures are conceptualized by activating the containment schema, and this is the mental ability to construct long sentences of human languages. 


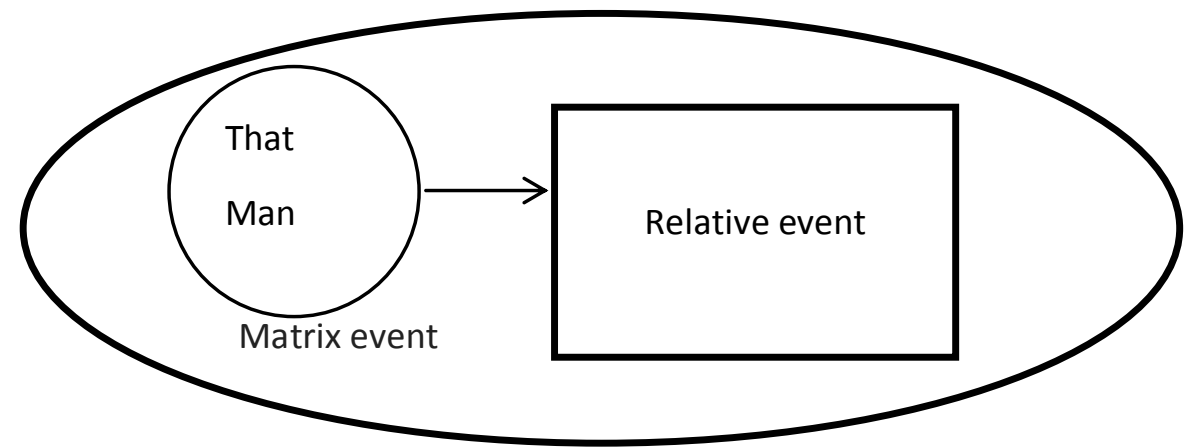

Figure1: containment schema of example(3)

\subsection{Construal}

The role of language, in cognitive approach, is not a direct modeling of elements of the world outside. In this perspective, different situations are not divided into smaller divisions, but a particular situation can be interpreted in a variety of ways. These various ways cause different perceptions (Lee, 2001: 2). Thus different ways of thinking about a particular phenomenon cause different construal of that phenomenon.

There is a profiled relation in each event and the human being, based on his interpretation of the event scene, conceptualizes that relation in his mind. Among the factors influencing the scene construal, are the prominence of components in a profiled relation by focusing attention on a specific component as well as selecting a special viewpoint for observing the scene.

There are usually two events in a relative situation: the first is an event that includes the subject modified and is called the matrix event, and the other one is an event modifying that subject and is called the relative event. When facing with a relative scene, the person, depending on his mental state and his construal of the scene, conceptualizes the relative structure. Thus, it is possible that from a constant situation, because of the prominence of a specific component or a different viewpoint, different construal is made, which will result in conceptualization and, ultimately, production of various relative structures.

\subsubsection{Attention Focus and Prominence}

Attention focus ,in fact, highlights an element in an expression and hence gives it a different interpretation. By changing the focus of attention from a component to another, in the profiled relations of a relative structure, the role of these components can sometimes be changed and the conceptualization of the relative structure will be influenced. Note the following:

(4-1) duste bæradæram ke ma ra be mehmani dæ?væt kærd to ra hæm mifenasæd.

$\begin{array}{cccc}\text { 3rdpsing } & \text { past } & \text { present,3rdpsing } \\ \text { friend brother my who us to a party invited you too knows. }\end{array}$

My brother's friend who invited us to a party knows you too.

In example (4-1),as shown in figures (2) and(3) ,the matrix event is "My brother's friend knows you too.". In the profiled relation in this event, "My brother's friend" is trajectory and "you" is landmark. The relative event is "(My brother's friend) who invited us to a party" in the profiled relation of which, trajectory is "My brother's friend" but landmark is "us." 

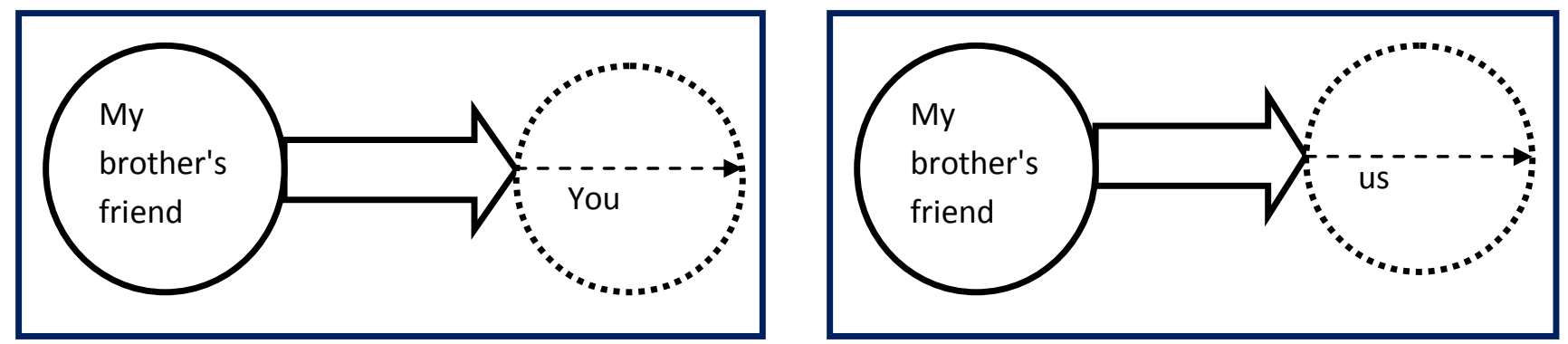

Figure2:Profiled relation in matrix event of example(4-1) Figure3:Profiled relation in relative event of example(4-1)

Now, if during the interpretation of the above scene, the focus of attention is changed from "you" to "us", the two landmarks of the two profiled relations will be changed with each other, and the two profiled relations, themselves, will change, as shown in figures(4) and(5), leading a different relative structure:

(4-2) duste bæradæram ke to ra hæm mifenasæd ma ra be mehmani dæ?væt kærd.

$\begin{array}{lcr}\text { 3rdpsing } & \text { present,3rdpsing } & \text { past } \\ \text { friend brother my who you too knows us to a party invited. }\end{array}$

My brother's friend who invited us to a party knows you too.

Matrix event: My brother's friend invited us to a party.

Trajectory: My brother's friend

Landmark: Us

Relative event: (My brother's friend) knows you.

Trajectory: My brother's friend

Landmark: you
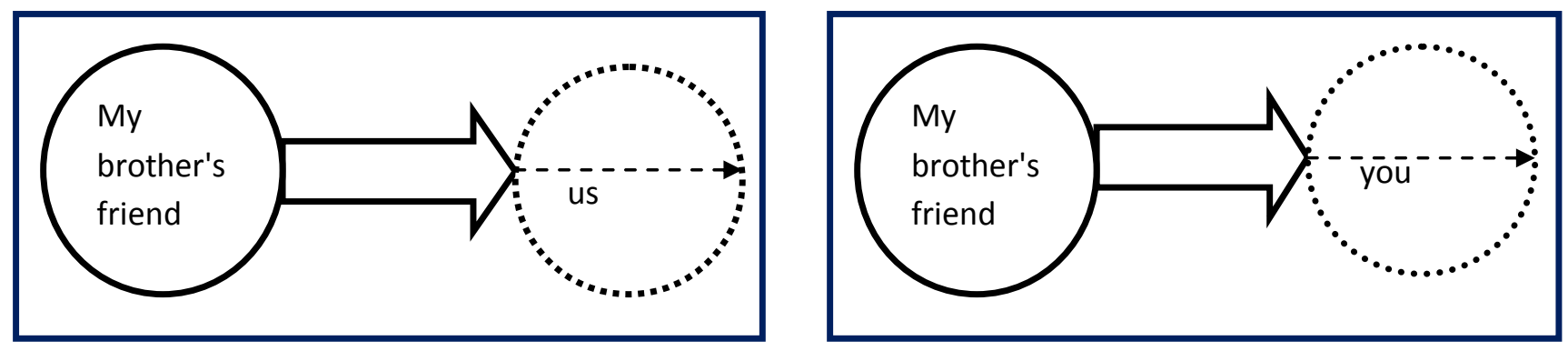

Figure4:Profiled relation in matrix event of example(4-2) Figure5:Profiled relation in relative event of example(4-2)

\subsubsection{Viewpoint}

Different viewpoints considered to describe a single position cause a meaning difference in descriptions of a single situation (Lee, 2001: 3-2). Paying attention to the viewpoint is, in fact, considering importance for the role of witnesses of a scene(Safavi,2008); Witnesses who choose a viewpoint and make different interpretations of the same scene. In the case of the relative scenes, the adoption of different viewpoints leads to different construal and, ultimately, different conceptualization.

For example, consider a situation where a blue pen is located on a notebook. In this situation, depending on viewpoint that the speaker chooses, two interpretations of the scene are obtained, which leads to the formation of two different relative structures. In the first interpretation, the viewpoint is on "pen" :

(5-1) xodkari ke ru dæftære mæn qærar daræd Pabi ?ææst.
3rdpsing
present,3rdpsing
present,3rdpsing

Pen which on notebook my is placed blue is. 
The pen which is placed on my notebook is blue.

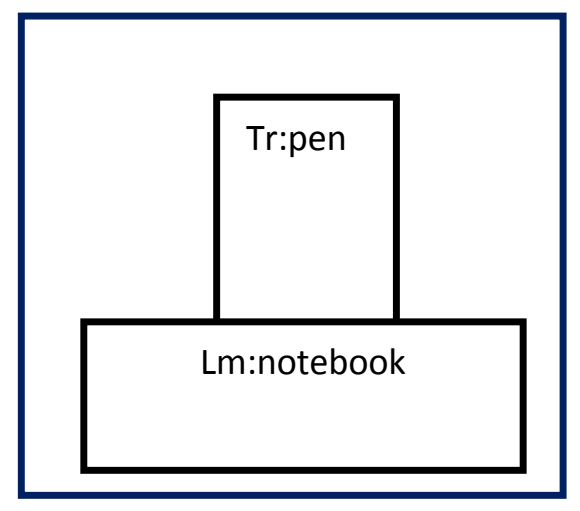

Figure 6 :Profiled relation in example(5-1)

In the second interpretation, viewpoint is on the "notebook":

(5-2) dæftæri ke zire xodkare Pabi qærar daræd male mæn ?æst. 3rdpsing present,3rdpsing present,3rdpsing

Notebook which under the pen blue is paced mine is.

The notebook which is placed under the blue pen is mine.

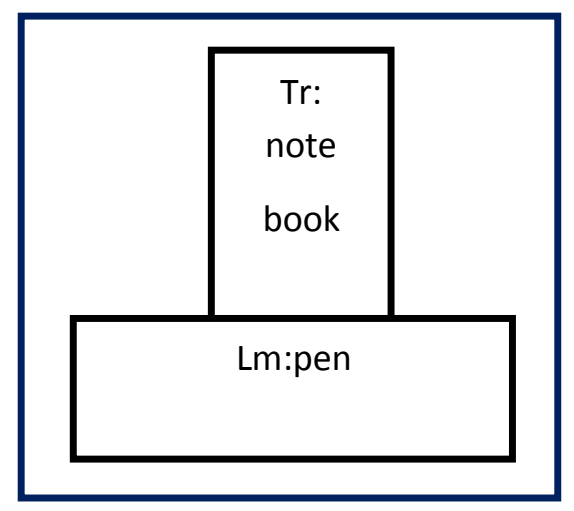

Figure7: Profiled relation in example(5-2)

As it was seen, in this case, by changing the viewpoint, from a constant situation, two relative structures are conceptualized and formed. Thus, it can be claimed that what modifies the head noun, , as a relative clause, is derived from the mental state of the speaker witnessing the scene, and this mental state itself is a result of the viewpoint that the speaker chooses to describe the scene in question, the attention that focuses on the components of the profiled relation, and the highlighting of a particular component.

\subsection{Symbolizing the Relative Structure}

One of the most prominent processes in CG considered by Langacker is profile which is the fundamental explanation of an event or a scene. Profile is, in fact, what an expression or a clause defines or refers to. Since there is always a profiled process in a given clause, one can claim that there is a kind of profiled relation among the components of a given clause. Among the most important components in a profiled relation, trajectory(Tr)and landmark(Lm) can be called. Trajectory and landmark are the first and the second most important contributors in a relation. In the perception of a relation, the more important participant getting the initial attention is trajectory and the other participant with less focus on it, is landmark.

On the other hand, according to CG, whatever conceptualized in the mind is symbolized in terms of the language units that are considered symbolic unit in this grammar, in other words, language has a symbolic behavior and the grammar with its symbolic structure uses the language units to symbolize concepts in speaker's mind. 
Symbolic units can be simple or compound. In CG, morphemes, words and even sentences are regarded as symbolic units. While, morphemes are simple units, compound words, phrases and sentences are compound units. In Langacker model, the units that have a compound symbolic structure are regarded as construct. (Evans \& Green, 2006: 5534). Therefore, one can consider a clause that is a compound symbolic unit as a construct. The writers believe since there are often two clauses found in a relative structure to symbolize matrix event and relative event, the relative structure can be considered as a macro construct consisting of two micro construct of matrix and relative. Thus, in following analysis, instead of the word "clause" the word construct will be used. Note the following example:

(6) hamsæræ[ hæman zæni ?æst ke Pindza kar mikonæd.

3rdpsing present3rdpsing present3rdpsing.

Wife his the same woman is who here works.

His wife is the same woman who works here.

The matrix construct "His wife is the same woman" symbolizes the matrix event in the relative structure and the relative construct "(his wife)works here", by modifying the subject "the same woman" symbolizes the relative event.

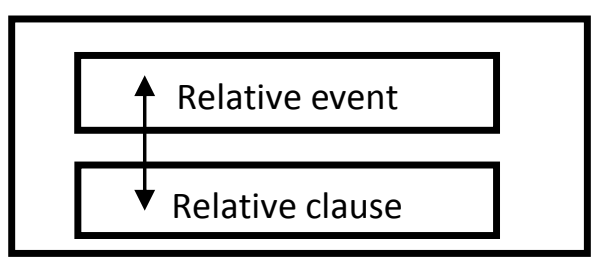

Figure 8: relative construct

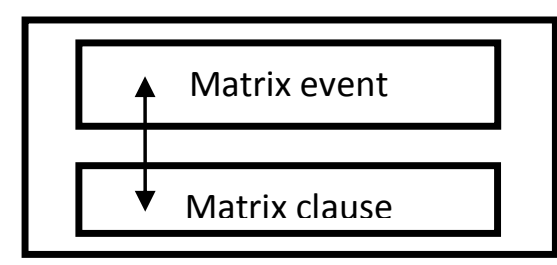

Figure 9: Matrix construct

\subsection{Relative Structure Analysis in Langacker Model}

Langacker $(2008,423-429)$ believes that during the modification of a nominal by a clause, there is a correspondence between the nominal referent and some participant in the process designated by the relative clause. That participant, has a semantic role in both the relative clause and the matrix clause containing the modified nominal. This role is sometimes the same in both clauses and sometimes the role of the participant in the two clauses, is different. On this basis, it is possible to divide the relative clauses. In the following section, based on the division of Murcia and Freeman, four types of subject-object, subject-object, object-subject and object-object Persian relative structures will be examined.

\subsubsection{Subject- Subject Relative Structures}

During the head noun modification process by a relative clause, a correspondence between the head noun roles in both clauses is made and the head noun referent is discovered in this way. In fact, by this correspondence, the head noun referent, which is present as a participant in the process, is identified. Since, in Langacker model, in each profiled relation existed in matrix and relative constructs, the main participants are trajectory and landmark, the head noun roles in Persian relative structures can be studied from this perspective:

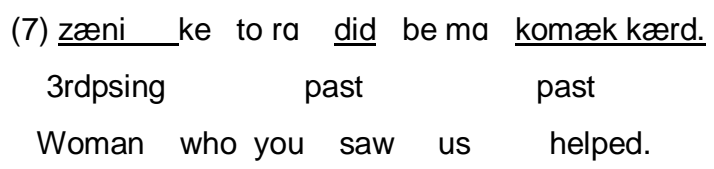

The woman who saw you helped us.

In the example (7), the head noun is "the woman" that in the profiled relation in matrix construct "The woman helped us" has trajectory role and in the profiled relation in relative construct "(The woman) saw you" has trajectory role too. Therefore, the desired correspondence takes place between the two trajectories (as shown in figure10). Accordingly, it can be claimed that in Persian Subject-Subject relative structures, the head noun plays the trajectory role in both matrix and relative construct, and the correspondence is made between these two trajectories. 


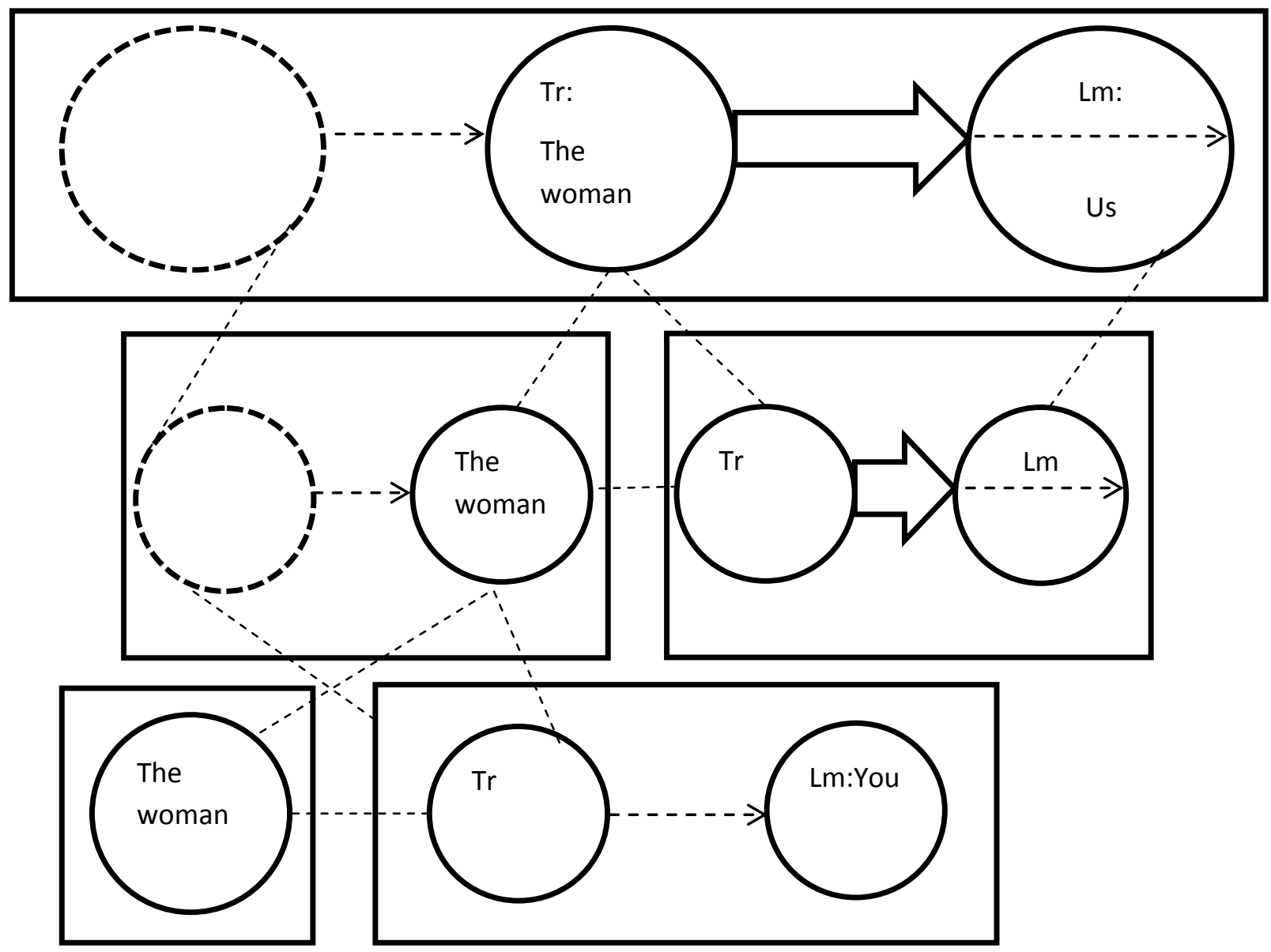

\subsubsection{Subject- Object Relative Structures}

Figure10: The correspondence in example (7)

In the subject-object structures, as shown in the example (8), the head noun plays the role of the trajectory in the matrix clause and the role of landmark in the relative clause. Consequently, the correspondence takes place between a trajectory and a landmark:

(8) ketabi ke xandæm mæra mote?æser kærd.

$$
\text { past past }
$$

Book which read me affected.

The book which I read affected me.

Head noun: The book

The role of head noun in matrix construct "The book affected me.": Trajectory

The role of head noun in relative construct "I read(the book)": Landmark

The correspondence is shown in figure (11). 


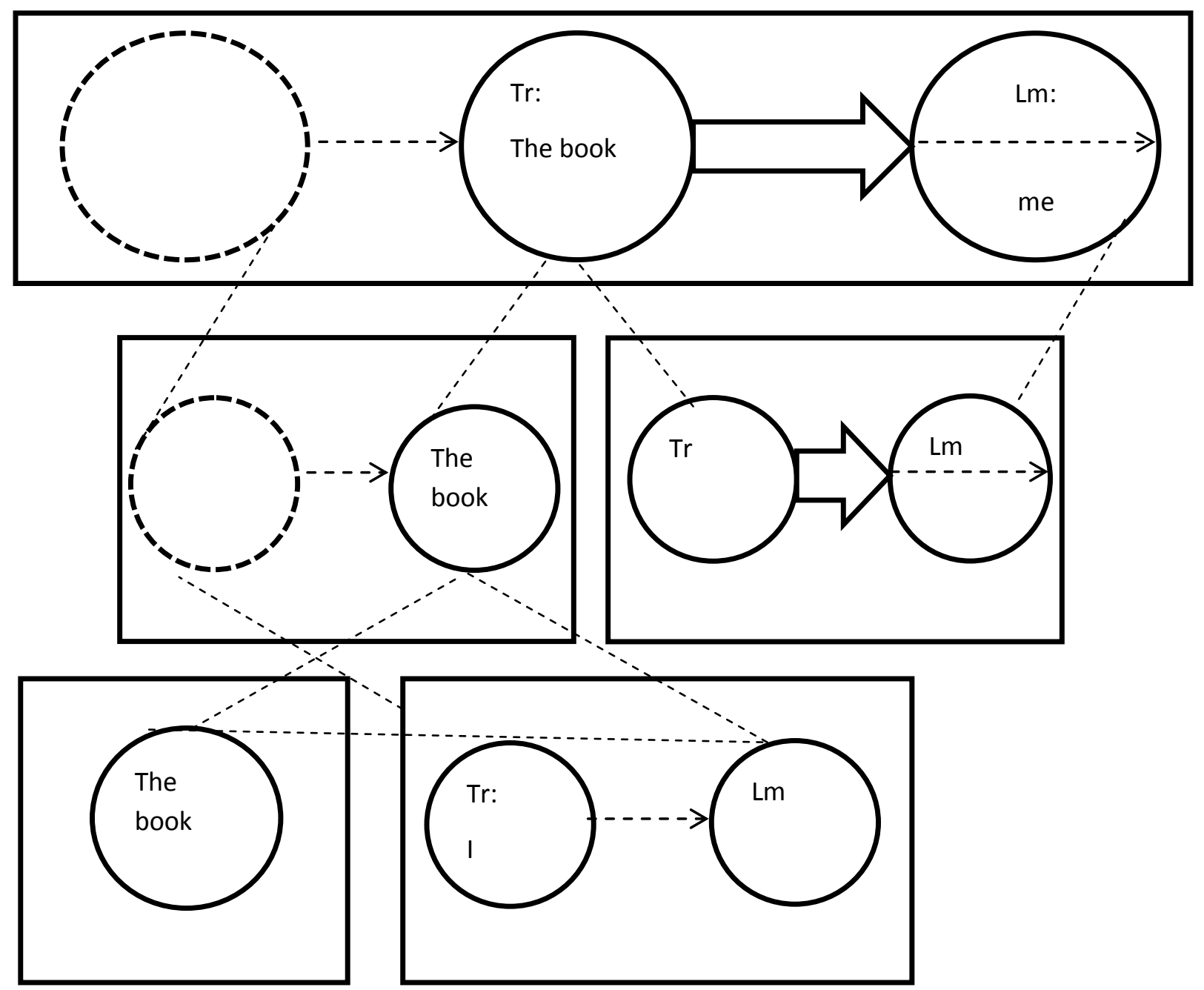

Figure11: The correspondence in example (8)

\subsubsection{Object-Subject Relative Structures}

Contrary to the previous section, in objects- subject relative structures the head noun plays a landmark role in the matrix clause and a trajectory role in the relative clause. Thus the correspondence takes place between a landmark and a trajectory:

(9) pedæræt ra didæm ke daft be Panha komæk mikærd.

past past

Fatheryour saw that was them helping.

I saw your father that was helping them.

Head noun: Your father

The role of head noun in matrix construct "I saw your father." :Landmark

The role of head noun in relative construct "(Your father)was helping them.": Trajectory

As it is seen in example (9),the correspondence is taken place between a landmark and a trajectory to discover the head noun. This correspondence is shown in figure (12). 


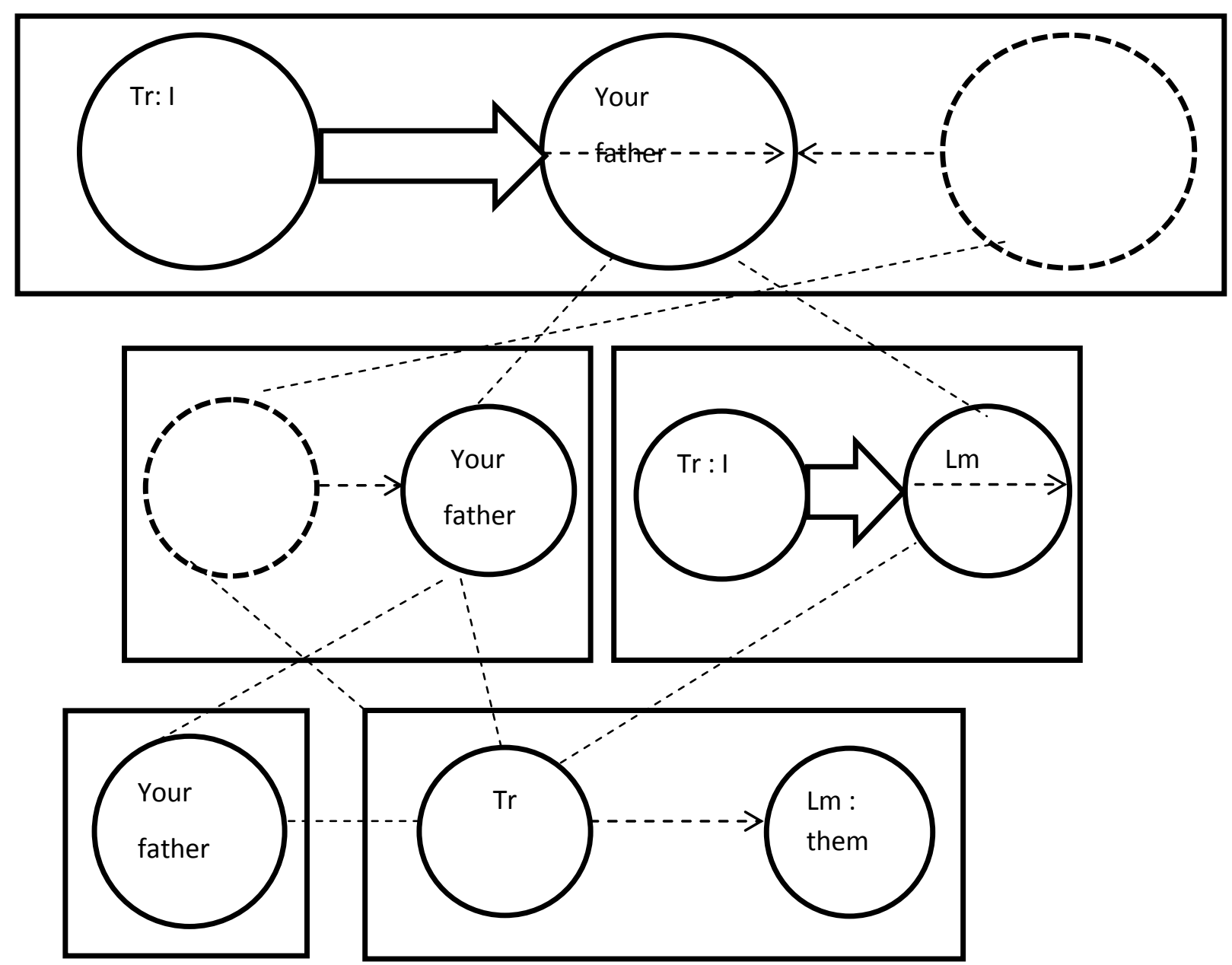

Figure12: The correspondence in example (9)

\subsubsection{Object-Object Relative Structures}

Finally, in the object-object relative structures, the head noun plays a landmark role in both the matrix and the relative clause. The correspondence is taken place between these two landmarks. Like the example(10):

(10) mærdi ra didæm ke to $\underline{\text { milenasi. }}$

$$
\begin{array}{llll} 
& \text { past } & \text { 2ndpsing ( Second person singular) present } \\
\text { man saw whom you know } &
\end{array}
$$

I saw a man whom you know.

Head noun: A man

The role of head noun in matrix construct "I saw a man." :Landmark

The role of head noun in relative construct "(A man) you know.": Landmark

The correspondence is shown in figure (13). 


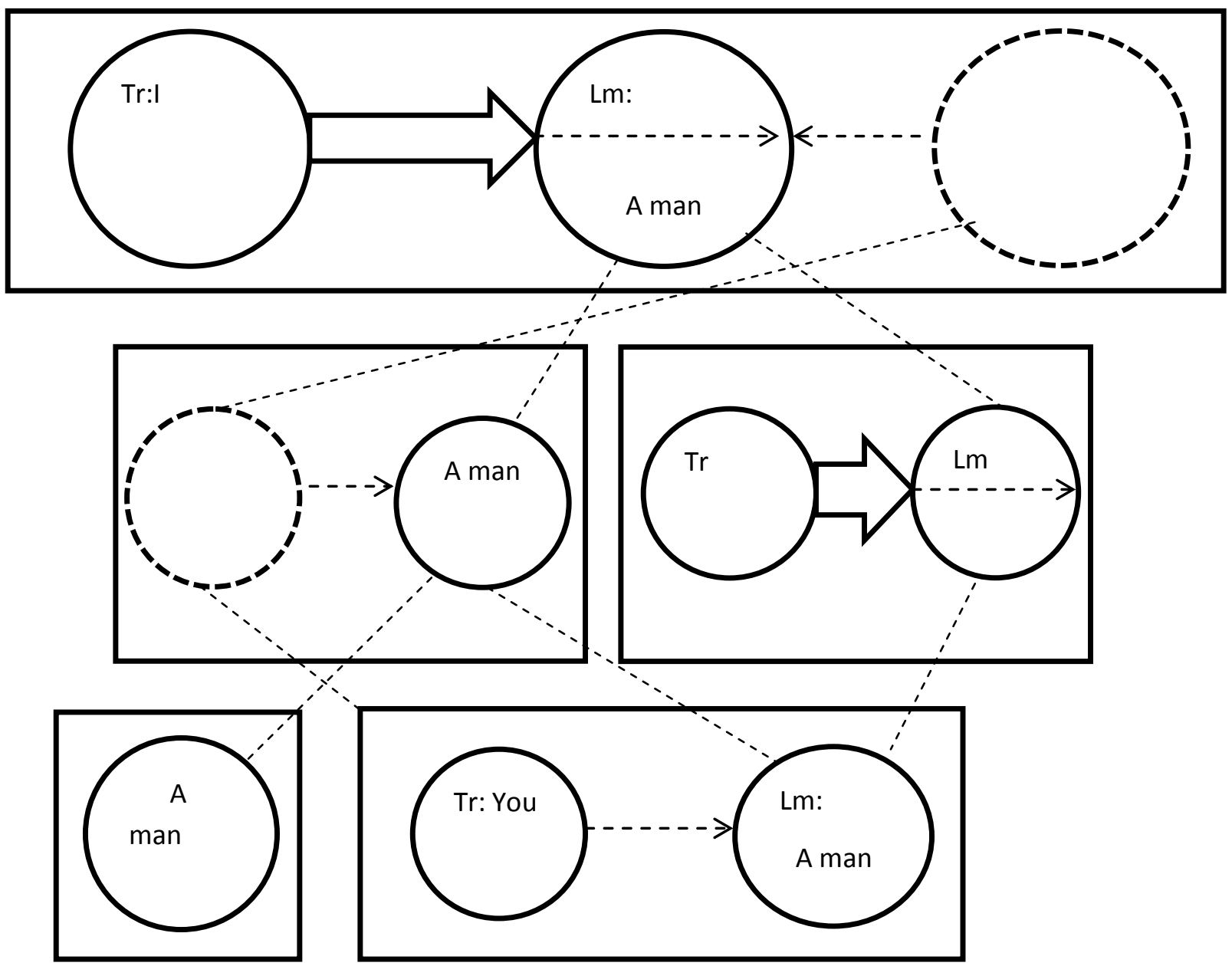

Figure13: The correspondence in example (10)

\subsection{Grounding}

Langacker (2008) refers the semantic difference between the restrictive and non-restrictive clauses to the way the head noun in these structures is grounded, the authors also believe that the difference between the restrictive and nonrestrictive clauses in Persian can be clarified the same way.

The profiled head noun, in a relative structure, is related to the scene and its participants by grounding. Unlike the restrictive clauses, the non-restrictive clauses do not play a role in determining the head noun referent. While the restrictive clause restricts the scope of the desired options, in non-restrictive structures, the profiled head noun is specified in the context, and its identification is independent of the attribute refered to it by the clause. Hence, in fact, it is grounding the head noun as a specific subject in a physical or linguistic context of relative structures that distinguishes non-restrictive clauses from the restrictive clauses. In Persian structures, the head noun can be grounded as a specific subject in different ways:

\subsubsection{Proper Noun}

Whenever, in a relative structure, the head noun is grounded as a proper noun whose referent is clear for the listener or reader, the clause following that, is a non-restrictive clause playing no role in identifying the head noun referent and only adds more information about it.

(11) tærane Pælidusti ke bazigære tævanmændi $\underline{\text { Pæst }}$ dær filme foru[ænde bazi kærd.

\section{3rdpsing}

Tarane Alidousti who actress capable

$$
\text { present, 3rdpsing }
$$

past

Tarane Alidousti, who is a capable actress, played in the movie "The salesman". 
Tarane Alidousti: The head noun grounded as a proper noun which is known and does not need to be identified.

\subsubsection{Known Subject}

In the structures where the head noun is grounded as a subject already known to the speaker and the listener, like the previous section, the relative clause doesn't have the identifying role and is a non-restrictive one:

(12) moælemane mæqtæ?e ebtedaji ke næq]e mohemi dær sævad Pamuzi dane

$$
\text { 3rdppl ( Third person plural) }
$$

Teachers level elementary who role important in education students

Pamuzan darænd , hoquqe kafi dærjaft nemikonænd.

$$
\begin{aligned}
& \text { Present } \\
& \text { Have salary enough get dont }
\end{aligned}
$$

Elementary school teachers, who play an important role in students education, do not get adequate salaries.

In example(12), "elementary school teachers" is a known subject and the relative clause "plays an important role in students education" has no role in identifying it and only provides more information.

\subsubsection{Identification in the Context}

If the head noun is grounded in such a way that its referent can be found in the physical context of the structure, there will be no need to identify its referent, and the role of the relative clause is to provide further information. In the example (13), in the house scene, the wife tells her husband:

(13)Pemruz pærde ajpæzxane ra,ke æz bæs joste jode digær rængi nædaræd,

Today curtain kitchen which from too much washing anymore anycolor doesn't have dobare [ostæm.

Past

again washed

Today, I washed again the kitchen curtain, which doesn't have any color any more from being washed too much.

Kitchen curtain: The head noun, which is grounded in the relative structure as an identified subject in the context.

\subsubsection{Definite Article}

Finally, the head noun may be grounded as a definite noun by the definite article and doesnt need to be identified so the role of the relative clause will be giving more information. In the example (14), the mother pointing to a book tells her son:

(14)?in ketab ra, ke bajæd dær tule term mixandi, bæraje jæbe Pemtehan gozafti.

$$
\text { past }
$$

present

This book which should during term study for night test put

This book, which you should have studied during the term, you want to study on the night before test.

\section{CONCLUSION}

In the present papers, it was determined that mental conceptualization and formation of Persian relative structure can be clarified in the cognitive approach by using the mechanisms of this approach. The viewpoint taken to describe a relative situation, or the change of attention focus from one component to another in a relative situation, can affect a relative situation construal, and eventually its symbolization as a relative structure. Also, the findings of the research show that Langacker model in analyzing the relative structures can be used to analyze Persian relative structures.

Another result from this research is that there are two kinds of relative clauses in Persian language: a restrictive and a non- restrictive ,and unlike many other languages, in Persian, these two kinds have formal differences. Additionally, all four categories of relative structures categorized by Murcia and Freeman, are found in Persian relative structures.

\section{REFERENCES}

1. Comrie, B. 1989. Language Universals \& Linguistic Typology. 2d ed Oxford: Blackwell.

2. Evans, V \& Green, M. 2006. Cognitive Linguistics: An Introduction .Edinburgh University Press Ltd. 
3. Gholamalizadeh, K. 1374. Structure of Persian language. Tehran: Ehya ketab.

4. Langacker, R. 2008 . Cognitive Grammar: A Basic Introduction. New York: Oxford University Press.

5. Lee, D .2001. Cognitive Linguistics: An Introduction. Oxford: Oxford University Press.

6. Murcia, M. and Freeman . 1999. The grammar book, Boston, Heinle \& Heinle.

7. Safavi,K. 2008. An Introduction to Semantics. Tehran: Islamic Art and culture Institute.

8. Whaley, L. J. 1997. Introduction to Typology: The Unity \& Diversity of Language. USA: Sage Publications.

\section{Biography of 1st Author}

Dr.Arsalan Golfam is associate profrssor in Linguistic department of Tarbiat Modarres University, Tehran,Iran.

\section{Biography of 2nd Author}

Dr.Alieh Kord Zaferanloo Kamboozia is associate profrssor in Linguistic department of Tarbiat Modarres University, Tehran,Iran.

\section{Biography of 3rd Author}

Dr.Ferdows Aghagolzadeh i is profrssor in Linguistic department of Tarbiat Modarres University, Tehran,Iran.

\section{Biography of 4th Author}

Sahel Khoshbakht Ghahfarrokhi is PhD linguistic student in Linguistic department of Tarbiat Modarres University,Tehran,Iran.

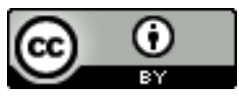

This work is licensed under a Creative Commons Attribution 4.0 International License. 